\title{
Acute flaccid paralysis: An approach to diagnosis
}

\author{
AK Yadav, P Bhattarai, B Aryal, PP Gupta, S Chaudhari, A Yadav \\ Department of General Practice and Emergency Medicine \\ B.P. Koirala Institute of Health Sciences, Dharan, Nepal
}

\begin{abstract}
Acute flaccid paralysis (AFP) is a common presentation to the emergency department and can present with paralysis of single to several limbs. Here is a case with AFP presented to highlight the approach to diagnosis. A 25 years male who recently returned from Malaysia presented to the emergency ward with acute onset of paralysis for two days which was progressive and was gradually involving the chest muscles as well. He had a history of loose mucoid stool since 4-5 days but there were no rashes or exanthema in the body. On complete examination, apart from diminished power in all the limbs, there were no other positive findings. He was started on expectant management and no other medications except multivitamins were prescribed. Patient was discharged home on patient party's request and on follow-up after 14 days, patient's had improved.
\end{abstract}

Keywords: Acute flaccid paralysis (AFP), emergency department

\section{Introduction}

Acute flaccid paralysis (AFP) is a clinical syndrome characterized by rapid onset of weakness, including (less frequently) weakness of the muscles of respiration and swallowing, progressing to maximum severity within several days to weeks ${ }^{1}$. It is preferable to use the term "paresis" for slight loss of motor strength and "paralysis" or "plegia" for severe loss of motor strength².

\section{Address for correspondence}

Dr. Ashok Kumar Yadav

Associate Professor

Department of GP \& EM

B.P. Koirala Institute of Health Sciences, Dharan

Email:dr_yadavashok@yahoo.com
The differential diagnosis of AFP varies considerably with age. No single operational clinical case definition of AFP having both high sensitivity and high specificity has emerged $^{3}$. Currently used methods have high sensitivity but specificity is lacking ${ }^{4}$.

\section{Case report}

A 25 years male, resident of Rajbiraj was admitted to the emergency department of BPKIHS, Dharan with complaints of inability to move bilateral lower limbs since two days which was acute in onset and gradually progressive to involve the proximal parts. His 
left hand was involved more than his right hand and was also complaining of inability to contract his chest properly while coughing. Patient also gave a history of loose stool since 4-5 days approximately, 2-3 episodes per day. Stool was mucoid and not blood mixed. There was no history of fever or rashes or no urinary or stool incontinence. The problem of inability to move the lower limbs started all of a sudden when he was returning home from his workplace. All of a sudden, his sandals had gotten off his feet and as he was trying to pick them up, he fell on the ground. There is no history of similar illness in the past or in the family or no documented chronic illness.

His general physical examination was within normal limit. On systemic examination, his higher mental function was intact and he was well oriented to time, place and person. His motor system revealed normal bulk in all the limbs but the tone was diminished in the bilateral lower limbs. Power was 2/5 on all the upper and lower limbs and there was no abnormal movements noted in the limbs. Coordination was normal among the limbs and there were diminished deep tendon reflexes in the bilateral upper and lower limbs. Babinski's test was negative bilaterally and pupils were normal in shape and size and reactive to light equally bilaterally. On sensory examination, his pain, touch, pressure, temperature, vibration sense and asteriognosis sensations were intact.

Cardiovascular and respiratory examinations revealed no significant findings.

Initial working diagnosis of acute flaccid paralysis? Viral neuropathy was made.
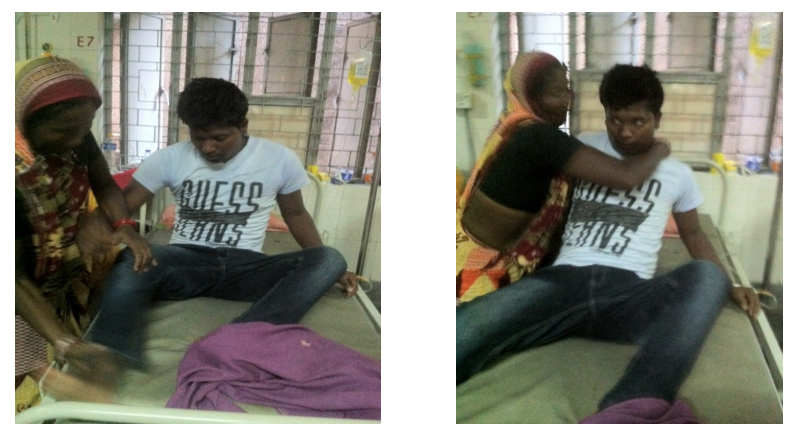

Fig. 1 and 2: Clinical photography
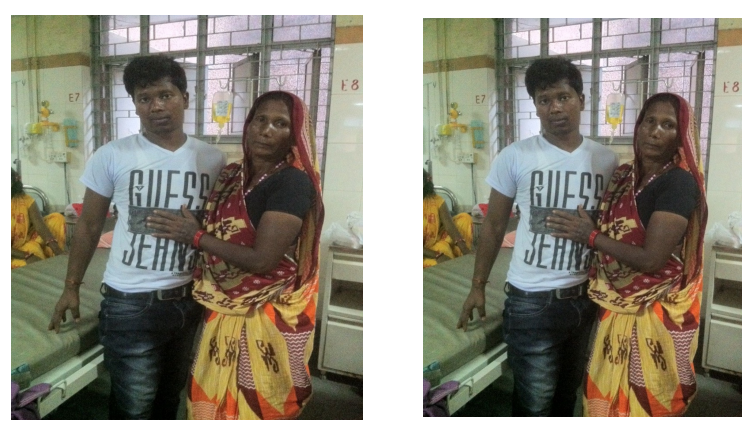

Fig. 3 and 4:Clinical photography

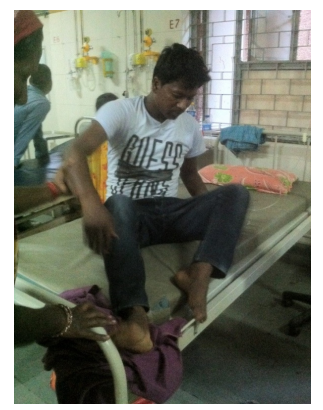

Fig. 5: Clinical photography

Management was started with intravenous fluids, Intravenous vitamin B complex and Pantoprazole $40 \mathrm{mg}$ i.v. in the emergency 
ward. His blood counts, random sugar, urea, creatinine, electrolytes, ECG, urine R/M/E were normal. His x-ray of the lumbosacral spine was also normal. An MRI of the lumbosacral spine revealed no abnormality.

In view of prodrome of fever and loose stool, a diagnosis of acute flaccid paralysis secondary to viral infection was made. However, he was kept on conservative management and discharged home. During discharge, physiotherapy was advised and an oral vitamin B-complex and a pantoprazole 40mg was given for 14 days and asked for follow-up.

During follow-up after 14 days, his symptoms had improved and the power in all the limbs were $4 / 5$ with normal superficial and deep reflexes.

\section{Discussion}

Each case of AFP is a clinical emergency and requires immediate examination. The clinical investigation of AFP is often limited by the existing health infrastructure and available resources ${ }^{1}$.

Flow chart for the workup of a weak patient

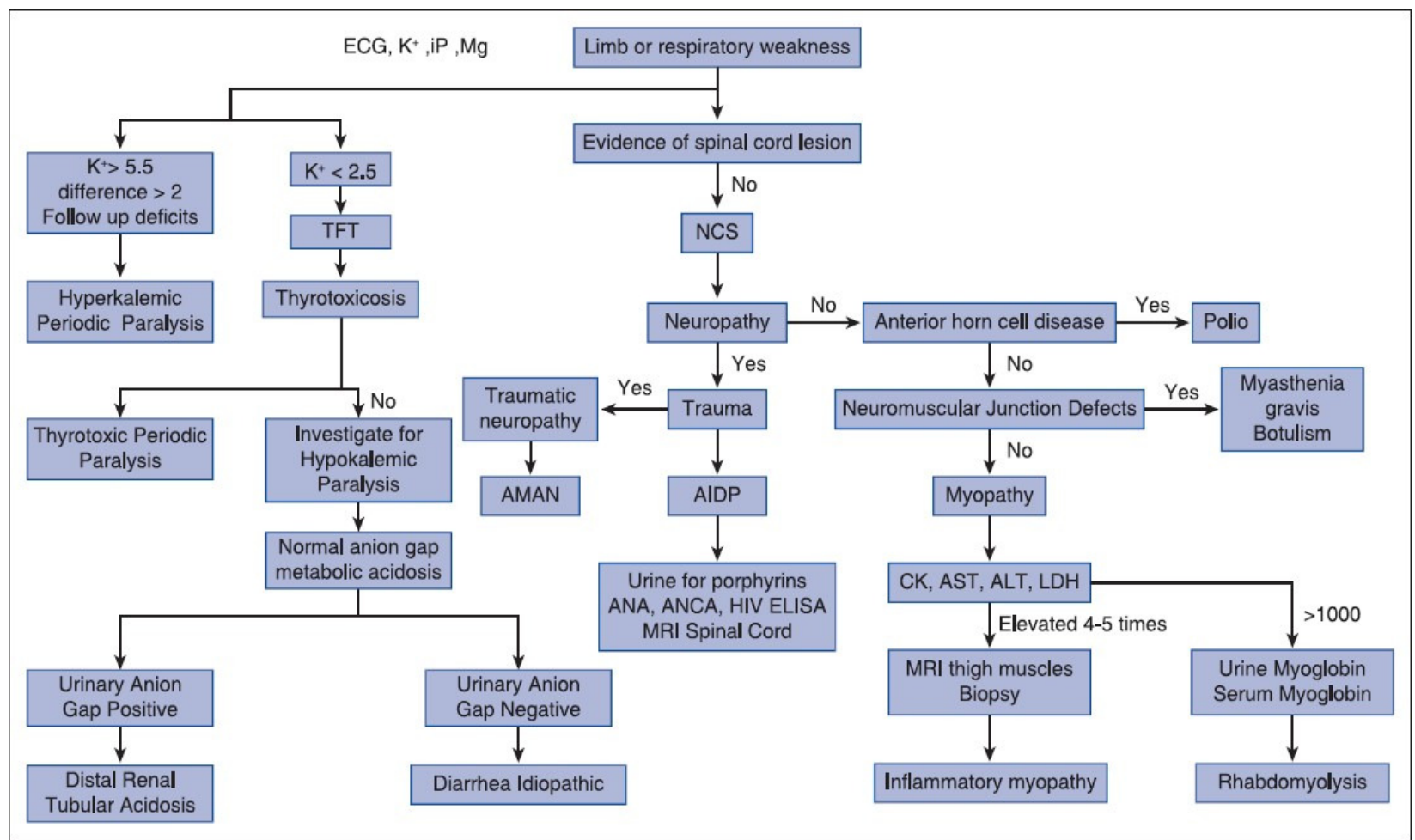

Common causes of AFP include Guillain Barre Syndrome, poliomyelitis, transverse myelitis, traumatic neuritis, postdiphtheric neuropathy and nonpolio enteroviral illness. 
D/D of AFP ${ }^{5}$ :

Muscle disorders:

Inflammatory myopathy

Periodic paralysis

Hypokalaemia

Infections

Neuromuscular junction disorders:

Myasthenia Gravis

Botulism

Eaton-Lambert syndrome

Neuropathies:

Guillain-Barre syndrome

Traumatic neuritis

Postdiphtheric neuropathy

Porphyria

Vasculitis

Anterior horn cell disorders:

Poliomyelitis

Nonpolio enteroviruses

Spinal cord disease:

Transverse Myelitis

Spinal Cord compression

Trauma

Since the control of poliomyelitis, GuillainBarre syndrome (GBS) has emerged as the most important cause of acute flaccid paralysis (AFP) in many developed countries $^{6}$. But in Nepal and developing nations, poliomyelitis still tops the list among the causes of AFP. Poliomyelitis presenting at adulthood is no so common. Hence,
Guillain Barre Syndrome (GBS) is the most common cause of AFP in adults. GBS, first described in $1916^{7}$ is regarded as an autoimmune disease that targets against peripheral nerves. About $50 \%$ of GBS patients have a history of acute infectious gastrointestinal or respiratory illnesses occurring 1- 4 weeks before onset.

\section{Clinical approach to patients with AFP $^{4,8,10}$}

A thorough clinical description of the symptoms should be obtained which includes fever, myalgia, distribution, timing, and progression of paralysis. The symptoms include gait disturbance, weakness, or troubled coordination in one or several extremities. Personal history (recent illness, erythema migrans in lyme borreliosis), timing of food and water consumption, exposure to chemicals (organic solvents), insect (tick) or snake bites, family history, vaccinations, and psychogenic problems, including dementia) should be interrogated. Trauma or spinal cord compression can be an obvious cause of AFP. Depending on the geographic region, the skin and scalp should be carefully inspected for ticks, stings from insects, spiders, or scorpions, and snake bites. Comprehensive neurologic examination, including assessment of muscle strength and tonus, deep tendon reflexes, cranial nerve function, and sensation (tactile sensation, vibration, and kinesthetic perception) is vital. 
Meningismus, signs of disordered central nervous system function (ataxia), or autonomic nervous system abnormalities (bowel and bladder dysfunction, sphincter tonus, neurogenic reflex bladder) should be kept in mind. Fasciculation is seen in anterior horn cell damage as well as demyelinating neuropathies. Electrophysiological studies determine the diagnosis and prognosis of lower motor neuron disease; nerve conduction velocity and electromyography studies, for example, can differentiate demyelinating neuropathies from axonal neuropathies. In currently or recently polioendemic countries, every case of AFP should be reported, regardless of its presumed etiology. Two stool specimens collected within 14 days after onset of paralysis, and virus isolation by a qualified laboratory is essential for diagnosis of polio myelitis. Serologic testing, isolation, and immunologic assays for enteroviruses, HIV, Herpesviridae (cytomegalovirus, Epstein-Barr virus, herpes simplex virus types 1 and 2, varicella-zoster virus), mycoplasma pneumoniae, campylobacter jejuni, and borrelia species, as well as a VDRL test can be done when indicated. For the diagnoses of immunologic or autoimmune disorders Antinuclear and antiGMj (glycolipid ganglioside-monosialic acid) glycoconjugate antibodies may be needed.

\section{Conclusion}

Acute flaccid paralysis is a very common condition encountered in the emergency ward. Systematic approach for diagnosis and treatment is very crucial for the proper management of the patients. However, in resource limited settings like ours, acute flaccid paralysis still poses a great challenge.

\section{References}

1. A. Marx, J. D. Glass, and R. W. Sutter, "Differential diagnosis of acute flaccid paralysis and its role in poliomyelitis surveillance," Epidemiologic reviews,. 2000:22; 298-316.

2. J. H. Growdon and J. S. Fink, "Paralysis and movement disorder," Harrison's principles of internal medicine. 2005: 11525.

3. V. Dietz, M. Lezana, C. G. Sancho, and R. Montesano, "Predictors of poliomyelitis case confirmation at initial clinical evaluation: implications for poliomyelitis eradication in the Americas," International journal of epidemiology, 1992: 21 ; 800806.

4. Z. Islam, B. C. Jacobs, M. B. Islam, Q. D. Mohammad, S. Diorditsa, and H. P. Endtz, "High incidence of Guillain-Barré syndrome in children, Bangladesh," Emerging infectious diseases. 2011:17; 1317. 
5. K. Marcdante, R. M. Kliegman, R. E. Behrman, and H. B. Jenson, Nelson essentials of pediatrics: Elsevier Health Sciences, 2010.

6. N. Yuki, T. Taki, F. Inagaki, T. Kasama, M. Takahashi, K. Saito, et al., "A bacterium lipopolysaccharide that elicits Guillain-Barré syndrome has a GM1 ganglioside-like structure," The Journal of experimental medicine.1993: 178; 17711775.

7. G. Guillain, J. Barré, and A. Strohl, "Sur un syndrome de radiculonévrite avec hyperalbuminose du liquide céphalorachidien sans réaction cellulaire. Remarques sur les caractères cliniques et graphiques des réflexes tendineux," Bull Soc Med Hop Paris. 1916: 40; 1462-70.

8. S. C. Singhi, N. Sankhyan, R. Shah, and P. Singhi, "Approach to a child with acute flaccid paralysis," The Indian Journal of Pediatrics. 2012:79; 1351-1357,.

9. J. Dematteis, "Guillain-Barre syndrome: a team approach to diagnosis and treatment," American family physician. 1996: 54; 197-200, 1996.

10. H. Azhary, M. U. Farooq, M. Bhanushali, A. Majid, and M. Y. Kassab, "Peripheral neuropathy: differential diagnosis and management," Am Fam Physician. 2010: $81 ; 887-892$. 